Musil-Forum 



\title{
Musil-Forum
}

Studien zur Literatur

der klassischen Moderne

\author{
Im Auftrag \\ der Internationalen Robert-Musil-Gesellschaft \\ herausgegeben von \\ Norbert Christian Wolf \\ und \\ Rosmarie Zeller
}

Band $31 \cdot 2009 / 2010$

De Gruyter 


\section{Redaktion: Harald Gschwandtner}

ISBN 978-3-11-026912-3

e-ISBN 978-3-11-027019-8

ISSN 1016-1333

Bibliografische Information der Deutschen Nationalbibliothek

Die Deutsche Nationalbibliothek verzeichnet diese Publikation in der Deutschen Nationalbibliografie; detaillierte bibliografische Daten sind im Internet über http://dnb.d-nb.de abrufbar.

(C) 2011 Walter de Gruyter GmbH \& Co. KG, Berlin/Boston

Satz und Druckvorlage: Martin Dieringer

Druck: Hubert \& Co. GmbH und Co. KG, Göttingen

$\infty$ Gedruckt auf säurefreiem Papier

Printed in Germany

www.degruyter.com 


\section{Inhalt}

Editorial .................. $\mathrm{V}$

Themenschwerpunkt: »Musil und die Fremdheit der Kultur«

Alexander Honold: Einleitung . . . . . . . . . 1

Nicola Gess: Expeditionen im Mann ohne Eigenschaften. Zum Primitivismus bei Robert Musil . . . . . . . 5

Birgit NÜBel: „ein dünner Dunst fremden Leibes«. Perversionen des Erkennens in Musils Essay Das Unanständige und Kranke in der Kunst . . . . . . . . . . . . . . . . . .

Robert Krause: "Man könnte die Geschichte der Grenzen schreiben«. Moosbruggers wildes Denken und die Kultur des Okzidents . . . . . . . . . . . . .

Axer Dunker: Soliman und Rachel/»Rachelle«. Die Konstruktion von Fremdheit und Identität in Robert Musils Der Mann ohne Eigenschaften . . . . . . . . . . . . . .

UlRICH Boss: Eine >bemerkenswerte Einzelheit . Arnheims phönikischer Schädel im Kontext antisemitischer Rassen-

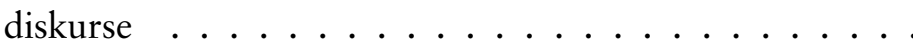

Walter Fanta: Ah, Fm: Doppelschichtung, unten jüdisch. Alles gilt, auch das Apokryph . . . . . . . . 84

Norbert Christian Wolf: Doktor Demant und Direktor Fischel. Zur >Alterisierung jüdischer Figuren in Roths $R a$ detzkymarsch und Musils Der Mann ohne Eigenschaften

Alexander W. Belobratow: Musil in Russland lesen. Eine Reise zu Ulrich mit dem Fürsten Myschkin, Ilja Oblomow und dem $>$ Kellerlochmenschen $<\ldots . . . . .$.

Alexander Honold: Fremdheit, Feindschaft. Österreichische Ökumene bei Musil und Handke . . . . . . . . 
BARBARA Thums: Fremdheit und Heterochronie in Robert Müllers Tropen . . . . . . . . . . . . . . 160

GÁbor Kerekes: Das Fremde im Werk Joseph Roths . . . 180

Abhandlungen

Peter Henninger $(\dagger)$ : Erlebnis, Dichtung und Kritik in Robert Musils Literarischer Chronik vom August I9I4 . . .

Regina Schaunig: Viribus unitis. Robert Musils Schreiben in kollektiver Anonymität . . . . . . . . . . . . 202

Heinz-Peter Preusser: Die Masken des Ludwig Klages. Figurenkonstellation als Kritik und Adaption befremdlicher Ideen in Robert Musils Roman Der Mann obne Eigen-

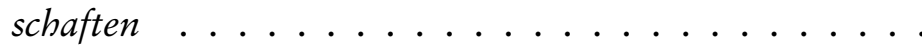

Rezensionen ................... 254

Anschriften der Beiträgerinnen und Beiträger . . . . . . . 281

Siglen . . . . . . . . . . . . . . . 283

Redaktioneller Hinweis . . . . . . . . . . . . . . . . 284

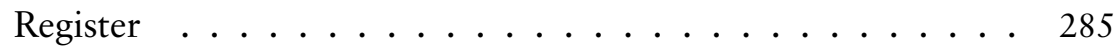




\title{
Nicola Gess
}

\section{Expeditionen im Mann ohne Eigenschaften}

\author{
Zum Primitivismus bei Robert Musil
}

ABSTRACT: The paper outlines Musil's reception of ethnological research and demonstrates its relevance for his literary texts. By analyzing the figure of Clarisse and the motives of madness, music, and language associated with her, it shows that the Mann ohne Eigenschaften draws on a complex engagement with the so-called "primitive«. The movement from expedition to self-experiment, already visible in Musil's early texts, is thus established as an essential structuring principle of the novel.

\section{1.}

Musils Rezeption ethnologischer Schriften hat in der Forschung bislang vergleichsweise wenig Beachtung gefunden. Zwar ist im Zusammenhang mit dem Essay »Ansätze zu neuer Ästhetik« oft auf seine Lévy-Bruhl-Rezeption eingegangen worden, jedoch wurde diese nur selten in Zusammenhang mit seiner Lektüre auch anderer ethnologischer und völkerpsychologischer Schriften gestellt, die vor allem in den frühen $20 e r$ Jahren $^{\mathrm{I}}$ stattfindet und sich in geringerer Intensität bis weit in die 3 oer Jahre zieht. ${ }^{2} \mathrm{Zu}$ nennen sind

1 Schraml weist darauf hin, dass Musils anthropologisches Interesse schon I9I3/I4 aufkommt, anlässlich eines Besuchs in Rom, bei dem er u. a. das anthropologische und ethnologische Institut, das Krankenhaus für Geisteskranke, das Typhus-Spital und die Affeninsel in der Villa Borghese besuchte. Nach diesen Besuchen häufen sich in Musils Aufzeichnungen Porträts von Personen und Tierbeschreibungen, in denen die "anthropologische Betrachtungsweise" auffällt (Wolfgang Schraml: Relativismus und Anthropologie. Studien zum Werk Robert Musils und zur Literatur der 2oer Jahre. München I994, S. 8I-93, hier S. 89).

2 Für ausführliche Auseinandersetzungen mit Lévy-Bruhl vgl. Renate Heydebrand: Die Reflexionen Ulrichs in Robert Musils Roman Der Mann obne Eigenschaften. Münster I966, S. I03-III. Willemsen geht ebenfalls in einem Kapitel auf Musils Interesse an Ethnologie ein und kontextualisiert Musils Lektüre Lévy-Bruhls mit Sprachkonzepten in Vico'scher Tradition sowie mit Jaenschs Eidetik-Forschung (Roger Willemsen: Das Existenzrecht der Dichtung. Zur Rekonstruktion einer systematischen Literaturtheorie im Werk Robert Musils. München I984, S. 286-297). Eine eingehende Lévy-Bruhl-Lektüre liefert auch Ritchie Robertson: Musil and the sprimitive mentality, in: Hannah Hickman (Hg.): Robert Musil and the literary landscape of his time. Salford I99I, S. I3-33. Allerdings gewinnt er daraus wenig Neues für die Auseinandersetzung mit Musil. Schraml bringt die vielleicht umfassendste Auseinandersetzung mit Musils ethnologischen Lektüren. Neben Lévy-Bruhl geht er im entsprechenden Kapitel seines Buches vor allem auf die Rezeption Müller-Lyers, ebenso Jaenschs und im Zusammenhang mit Jaenschs entwicklungpsychologischen Überlegungen auch auf jene von 
hier neben Lévy-Bruhls Das Denken der Naturvölker (in dt. Übersetzung I923) vor allem Müller-Lyers Phasen der Kultur (I9I5/1908), Jaenschs Die Völkerkunde und der eidetische Tatsachenkreis (1923) und Kretschmers »Entwicklungsgeschichte der Seele« aus der Medizinischen Psychologie (I922), die Musil alle 1922/23 exzerpierte und in eigenen Publikationen erwähnte, sowie Texte von Hornbostel zur Dichtung und von Cassirer zu den Sprachen der sogenannten Naturvölker, die Musil Anfang der zoer Jahre rezipierte. Hinzu kommt eine Vielzahl einschlägiger Titel, die Musil sich ebenfalls 1923 notierte, zu denen aber keine Exzerpte vorliegen, z. B. die Schriften Preuss' (u. a. Die geistige Kultur der Naturvölker [19I4]), Thurnwalds Forschungen auf den Salomo Inseln und dem Bismarck Archipel (I912), Vierkandts Kulturvölker und Naturvölker (1896) sowie eine Reihe von Texten zu Felszeichnungen der Naturvölker. ${ }^{3}$ Musil verfährt in der Lektüre der genannten Schriften selektiv. Er interessiert sich (I) für Wahrnehmung, Vorstellung und Denken der sogenannten Naturvölker, genauer für Phänomene der Partizipation, für das eidetische Vermögen und für das affektgesteuerte Denken, (2) für Eigentümlichkeiten der Sprachen und des Sprachverhältnisses, nämlich für die Anschaulichkeit der Sprachen, das Wörtlichnehmen figurativer Sprache und die Partizipation des Wortes an seinem Gegenstand, (3) für die magische Funktion der Kunst der sogenannten Naturvölker, d. h. ihre Ausrichtung auf Herstellung statt Darstellung des Gewünschten. ${ }^{4}$

Groos' Spiele der Tiere ein. Dabei interessiert sich Schraml besonders für die "appetitive» Zeichnung des "Archaischen « bei Musil (Schraml: Relativismus, S. I27-I39). Florence Vatan (Robert Musil et la question anthropologique. Paris 20oo, S. 73-88) weist in einem Kapitel ihres Buches auf Affinitäten der Figuren Agathes, Moosbruggers und Clarisses zum Denken der sogenannten »Primitiven« bei Lévy-Bruhl hin sowie auf Kollektivrituale, mit denen Musil die Affinitäten seiner Gesellschaft zur »'enfance de la civilisation" (S. 8o) offen legt, und auf Totem- und Tabu-Figuren. Schließlich setzt sich auch Genese Grill: The »Other« Musil: Robert Musil and Mysticism, in: Philipp Payne, Graham Bartram, Galin Tihanov (Hg.): A Companion to the Works of Robert Musil. Rochester, London 2007, S. 333-354, mit Frazer, Hornbostel und Lévy-Bruhl auseinander, um sie als wichtige Quellen für Musils Interesse an "mystical ritual activity« und eines »religious origin of art« $(\mathrm{S} .337,338)$ zu lesen.

3 Für die vollständige Literaturliste vgl. KA/Transkriptionen/Heft 2I/II4. Auf der Heftseite davor findet sich eine weitere Literaturliste (entnommen aus Klages Vom kosmogonischen Eros) zur Mythenforschung, insbesondere zu antiken Kulten und Mysterien. Hinzu kommen weitere Texte, die weder im Rahmen von Literaturlisten noch von ausführlichen Exzerpten auftauchen, sondern nur kurz erwähnt werden, wie z. B. im gleichen Heft Alexander von Humboldts Reise in die Äquinoktialgegenden (KA/Transkriptionen/Heft 2I/53), oder in KA/Transkriptionen/Mappe VII/II/36 »Frobenius Afrikabuch« (vgl. KA/Transkriptionen/ Mappe IV/2/I78).

4 Darauf gehe ich ausführlich im Musil-Kapitel meines derzeit im Entstehen begriffenen Buches Figurationen primitiv(istisch)en Denkens. Zur Verschränkung von Ethnologie, Psychologie und Literatur im frühen 20. Jahrhundert ein. 
Musils Faszination für die Ethnologie schlägt sich auch in seinen fiktionalen Texten der frühen zoer Jahre nieder. Nach dem Vorbild von Romanen wie Müllers Tropen oder von Novellen wie Jensens Wälder, die Musil beide schätzte, ${ }^{5}$ schreibt er Expeditionserzählungen, wie z. B. Grigia oder das unvollendete Projekt »Land über dem Südpol«. In beiden fällt die Bewegung vom Fremden zum Eigenen auf, die mit einer Bewegung von der Expedition zum Selbst-Experiment einhergeht. Das Prosaprojekt »Land über dem Südpol «, das in seinen Anfängen bis I9ı I zurückreicht und dessen letzte Notizen von I929 stammen, erinnert in seiner Anlage zum Beispiel an Kubins phantastischen Roman Die andere Seite. In beiden Fällen geht die Faszination von der utopischen Andersartigkeit der fremden Gesellschaft aus, in die die Expediteure reisen. Bei Musil handelt es sich dabei um eine Kultur, in deren Zentrum Humanexperimente stehen. Eines davon skizziert Musil besonders ausführlich. Mithilfe biologischer Manipulation werden hier tierische Verhaltensweisen im Menschen reaktiviert: »Man läßt Menschen alle Tiere durchlaufen. Mit biologischen Methoden. Sie sättigen sich, reagieren $a b \ll{ }^{6}$ Dabei interessieren Musil vor allem die bizarren Begattungsriten von Amphibien. Aus Brehms Tierleben macht er sich umfangreiche Notizen, insbesondere zu solchen Riten, die die Tötung des Sexualpartners oder auch eines dritten Tiers beinhalten. Daran schließen sich Phantasien über Parallelen im menschlichen Verhalten, z. B. Sexualverbrechen oder monströse Mutationen menschlicher Körper an.

Was sich nach einer Kultur der perversen Lüste anhört, ist jedoch tatsächlich eine Kultur des Experiments: »Eine Welt regiert von wenigen, die in einer Art Kloster [der Geistigen] leben. Welt, eingeteilt in Experimentalfelder. «7 »Menschen, die alle intellektuell möglichen Konstellationen leben. [...], ihre ganze Energie liegt im Seelischen $«{ }^{8}$ Die Menschen, um die es hier geht, sind als Experimentatoren und Versuchsobjekte gespalten in eine geistige und eine körperliche Existenz, identifizieren sich aber allein mit der ersteren. Denn sie sind kaum involviert in das Geschehen, das sie doch phy-

5 Musil erwähnt beide Texte im Nachruf auf Müller. Müller hat auch andere Texte dieses Genres verfasst, z. B. die Novelle Das Inselmädchen, die Musil im Nachruf ebenfalls nennt, ohne jedoch näher auf sie einzugehen. I92I bittet Musil in einem Brief an Arne Laurin (I2.03. I92I) diesen darum, einem Rezensenten eine Reihe Bücher schicken zu lassen, darunter mehrere Titel dieses Genres, z. B. Douglas Mawson, Leben und Tod am Südpol, Gauguin, Briefe aus der Südsee, Graf Vay de Vaya, Nach Amerika im Auswandererschiff, J. V. Jensen, Das verlorene Land, Ejnar Mikkelsen, Sachawachiak, der Eskimo (vgl. Br I, S. 22I). Dies ist als weiterer Hinweis darauf zu verstehen, dass Musil mit diesem Genre durchaus vertraut oder mindestens daran interessiert war.

$6 \mathrm{KA} /$ Transkriptionen/Heft 2I/I34.

7 KA/Transkriptionen/Mappe IV/2/178.

$8 \mathrm{KA} /$ Transkriptionen/Mappe IV/2/5. 
sisch betrifft. Ihr einzig wahres Anliegen ist ihre Forschung: "Sie selbst sind ohne Leidenschaften [...]. Sie haben schon Sympathien udgl. aber sie sind sich der Herkunft bewußt (aus der Tierreihe) u. behandeln sie nicht ernst. Vielleicht ist ihr Leben monoton, aber es wird von einem ungeheuren $\mathrm{Ar}$ beitsdrang ausgefüllt «. ${ }^{9}$ Deutlich verschiebt sich hier die Bewegung von der Expedition ins Fremde zu einem Experiment mit dem Ent-Fremdeten im Eigenen, an dem der Expediteur partizipiert. Bezeichnenderweise handelt es sich bei den Bewohnern des fremden Planeten auch nicht um Fremde, sondern um ent-fremdete Eigene, nämlich ausgewanderte Deutsche.

Auch die Novelle Grigia handelt von der Expedition eines zivilisationsmüden Wissenschaftlers in eine fremde Kultur, allerdings nicht in eine Science-Fiction-Welt, sondern in eine vorzivilisatorische Gesellschaft. ${ }^{\text {IO }}$ Wie in »Südpol« zeigt sich auch in dieser Novelle schnell, dass man es bei den Fremden eigentlich mit einem entfremdeten Eigenen zu tun hat: Abermals handelt es sich um Nachfahren deutscher Auswanderer. Außerdem gerät, wie in den Erzählungen Jensens oder Müllers, die Expedition schnell zum SelbstExperiment, wenn ihre Teilnehmer sich auf unterschiedliche Weise und in post-zivilisatorischer Verzerrung das vermeintlich "primitive "Verhalten der Bergbewohner zu eigen machen.

3.

Meine These ist, dass auch der Mann ohne Eigenschaften eine komplexe Beschäftigung mit dem sogenannten »Primitiven« enthält und sich die Bewegung von der Expedition zum Selbst-Experiment als Konstruktionsprinzip des Romans ausmachen lässt. ${ }^{\mathrm{II}}$ Damit ist nicht nur der viel beschriebene Laborblick gemeint, der Ulrich, den Erzähler und auch Musil in seinen Bemerkungen zum Roman auszeichnet. ${ }^{12}$ Was in den frühen Erzählungen auf

9 KA/Transkriptionen/Heft II/75-76.

10 Dass es sich um eine vorzivilisatorische Gesellschaft handelt, zeigt überzeugend Schraml: Relativismus, S. I40 ff.

11 Bei der Rede vom »Primitiven«, wie sie im ethnologischen Diskurs um I9oo gang und gäbe war, handelt es sich um eurozentristische Konstruktionen des Fremden, mit hochproblematischen Implikationen, wie z. B. die Subsumierung verschiedenster Kulturen und ihrer Errungenschaften unter das Label des »Naturvolks«; oder die evolutionistische Annahme einer niedrigen Entwicklungsstufe, der die europäische Gesellschaft als höchste Entwicklungsstufe übergeordnet wird. In diesem Aufsatz wird dieser Wortgebrauch zwar aufgegriffen, weil es mir unter anderem um die kritische Rezeption dieses Diskurses bei Musil geht; gleichzeitig distanziere ich mich aber davon, indem ich von "primitivistisch « spreche oder ein "sogenannt" vor die entsprechenden Ausdrücke setze, um den Konstruktcharakter und die Zugehörigkeit $\mathrm{zu}$ einem europäischen Imaginationsraum zu betonen.

12 Ihm widmet sich in Bezug auf den Nachlaß zu Lebzeiten ausführlich Thomas Hake: "Gefühlserkenntnisse und Denkerschütterungen«. Robert Musils Nachlaß zu Lebzeiten. Bielefeld I998, S. I22-I6I; vgl. auch das jüngst erschienene Buch von Andrea Pelmter: »Experimen- 
thematischer Ebene verhandelt wird, kehrt im Roman auf einem hohen Reflexionsgrad und strukturell gewendet zurück. Die frühen Erzählungen offenbaren das »Primitive« klar als Konstruktion - schließlich gibt es schlichtweg kein Land über dem Südpol. Deutlich wird, dass es dabei um ein entfremdetes Eigenes geht, nämlich um ein alternatives Weltverhältnis. Musil und sein Alter Ego Ulrich interessieren sich im Roman deshalb nicht mehr für das ethnologisch Fremde, sondern für das Fremde in der eigenen Kultur: »[U]nsre heimischen Primitiven sind uns fremder als die der Südsee. ${ }^{\mathrm{I} 3}$ Entsprechend tritt an die Stelle der Ethnologie die Psychologie, die aber wohlgemerkt durch den ethnologischen Blick inspiriert bleibt. Das heißt auch, dass die Fremdheiten der eigenen Kultur weiterhin als "primitiv" codiert werden. Mit dieser Codierung ist erstens eine Genealogie verbunden - dieses Fremde im Eigenen hat uralte Wurzeln; zweitens eine anthropologische These - es handelt sich dabei um ein genuin menschliches Vermögen; und drittens eine negative Bewertung von Regressionsbewegungen. Das aus der Expedition sich ergebende Selbst-Experiment unterscheidet sich vom Laborblick außerdem dadurch, dass die Experimentatoren selbst involviert sind, ob im Sinne einer Veränderung des Denkens, wie Ulrich, oder im Sinne einer Veränderung des Schreibens, wie Musil. Beide partizipieren, das wird zu zeigen sein, an den Primitivismen, die der Roman reflektiert.

Im Folgenden möchte ich zunächst die erste Hälfte meiner These, die Expedition ins Primitivistische, die der Roman vornimmt, anhand der Figur der Clarisse erarbeiten. In den Entwürfen des Romans finden sich zahlreiche Hinweise, die eine Verwandtschaft der geisteskranken Clarisse mit den sogenannten »Primitiven « nahelegen. In einem Entwurf aus der Zwillingsschwester-Phase des Romans schreibt Clarisse während ihrer ersten Internierung einen Text, indem sie darüber reflektiert, dass in früheren Jahrhunderten »religiös Erweckte« wie Franz von Assisi die Möglichkeit zu einem normalen Leben hatten, während sie heute als Manische in Anstalten versteckt werden (MoE, S. I734). In einer Entwurfsstufe der Spion-Phase stellt diese Überlegungen noch Musil selbst an und ergänzt sie wie folgt: »[D]as, was heute nur noch in einem Gebirgsdorf möglich ist [...] [, konnte] damals in einem Zentrum der Kultur sich ereignen" (MoE, S. I80I). Die geisteskranke Clarisse wird so als Verwandte Grigias, der bäuerlichen Nachfahrin einer vor-zivilisatorischen Kultur kenntlich gemacht. In einem anderen Entwurf aus dieser Phase beschreibt Musil den psychischen Zustand Alice Donaths, des Vorbilds Clarisses, auch als ein »Wiederaufleben mittelalterlicher Seelenzustände in neuer Fassung " (MoE, S. I794). Weitere Hinweise auf die Verwandtschaft Clarisses mit den sogenannten »Primitiven« liefern zum Beispiel ihre »Entdeckungen« in den Entwürfen der Insel-Kapitel, dass die »untergegangenen

tierfeld des Seinkönnens« - Dichtung als »Versuchsstätte«. Zur Rolle des Experiments im Werk Robert Musils. Würzburg 2008.

13 Robert Musil: Bücher und Literatur, in: GW 8, S. II70-II80, hier S. II7I. 
[...] phantastischen Wälder der Carbonzeit « heute »als Psychisches wieder frei« werden (MoE, S. 1755; siehe auch MoE, S. I795). Der Erzähler vergleicht entsprechend die Geräusche der Inselnacht mit der Aufregung in einem »Negerdorf, das zum Tanz antritt « (MoE, S. 1755). All diese Hinweise lassen es nur folgerichtig erscheinen, dass Musil seinen Roman noch 1932 tatsächlich als eine Expedition bezeichnet: »Dieses Buch ist [...] eine geistige Expedition und Forschungsfahrt $\ll{ }^{\mathrm{I} 4}$

\section{4.}

Den Hinweisen der Entwürfe auf Clarisse als Figuration des sogenannten "Primitiven « möchte ich anhand von drei Motivkomplexen genauer nachgehen. Erstens: Primitivismus in Manie und Schizophrenie. Die Psychopathologie des frühen 20. Jahrhunderts versteht bestimmte psychische Störungen, vor allem die Schizophrenie, als Regressionen auf das phylogenetische Stadium des sogenannten »primitiven Denkens«, das ich im Folgenden, um es als europäisches Konstrukt zu kennzeichnen, nur noch als primitivistisches Denken bezeichnen werde. Stellvertretend für viele andere sei für diese Regressionsthese nur Kretschmer zitiert: »Im schizophrenen Denken [...] [können] weite Zusammenhänge des primitiven Weltbildes wieder vor uns lebendig [werden] « ${ }^{15}$ Freud verspricht sich daher von der Psychoanalyse nicht nur Aufschluss über psychische Krankheiten, sondern zugleich über die mentalen Operationen der prähistorischen Menschheit: "[Die] Neurose [hat uns] mehr von den seelischen Altertümern bewahrt [...], als wir vermuten konnten, so daß die Psychoanalyse einen hohen Rang unter den Wissenschaften beanspruchen darf, die sich bemühen, die ältesten und dunkelsten Phasen des Menschheitsbeginns zu rekonstruieren. ${ }^{16}$ Folgt man Freud, so ist die angemessene Wissenschaft zur Erforschung des sogenannten »Primitiven« also gar nicht unbedingt die Ethnologie, sondern vielmehr die Psychologie, die anhand von psychischen Störungen die Geheimnisse des primitivistischen Denkens erkundet.

Musil greift diese Bewegung auf, indem er Clarisse als manische Figur mit schizophrenen Einschlägen kennzeichnet. In den zoer und frühen zoer Jahren beschäftigt sich Musil intensiv mit Bleulers Lehrbuch der Psychiatrie sowie mit Kretschmers Medizinischer Psychologie und mit Oesterreichs Die Phänomenologie des Ich in ihren Grundproblemen. Alle drei Texte beeinflussen deutlich die Konstruktion der Clarisse-Figur in den Kapitelentwür-

$14 \mathrm{KA} /$ Transkriptionen/Mappe II/I/65.

15 Ernst Kretschmer: Medizinische Psychologie. Leipzig I922, S. I37.

16 Es handelt sich um einen I9I9 ergänzten Zusatz zur Traumdeutung; in: Sigmund Freud: Studienausgabe. Hg. v. Alexander Mitscherlich. Bd. 2: Die Traumdeutung. Frankfurt a. M. I996, S. 524, siehe auch S. 540. 
fen »Frühspaziergang «, "Waffenstillstand « und »Hermaphrodit«, in denen Clarisses Manie - Musil spricht vom »sich ankündigende[n] Manisch[-] Aktive[n] ${ }^{17}$ - zum endgültigen Ausbruch kommt und zum Dauerzustand wird. ${ }^{18}$ Dabei werden viele Eigenarten Clarisses, die bereits in älteren Kapiteln und Kapitelentwürfen entwickelt wurden, wieder aufgegriffen, um nun gewissermaßen zu Symptomen ausgearbeitet und ihrer impliziten Diagnose als Manie mit schizophrener Tendenz zugeführt zu werden. Ich beschränke mich hier darauf, die Merkmale zu nennen, die das manisch-schizophrene mit dem primitivistischen Denken verbinden, wie Musil es in seinen ethnologischen Exzerpten skizziert hat. Diese Verwandtschaft betrifft die asyntaktischen Bildserien, die das für die Manie typische ideenflüchtige Denken Clarisses auszeichnen; ebenso die eidetischen Anschauungsbilder, aus denen sich die Bildserien zusammensetzen. Ihre Zusammensetzung gehorcht, wie das primitivistische Denken auch, dem Prinzip der Assoziation auf der Basis äußerer Ähnlichkeiten oder Kontiguitäten. Häufig folgt Clarisses manisches Denken aber auch einer katathymen Logik, die es ebenfalls mit dem primitivistischen Denken teilt. Mit dem wunschgesteuerten, dereistischen Denken verweisen Bleuler, Musil und Clarisse schließlich selbst auf die Verwandtschaft des schizophrenen Denkens mit dem mythischen. Bleuler schreibt: »In diesen Formen geht die Dereation bis zur Auflösung der gewöhnlichsten Begriffe; [...] Apollo wird in mehrere Persönlichkeiten gespalten [...], obgleich er für gewöhnlich ein Mann ist, kann er auch eine Frau sein «. ${ }^{19}$ Musil greift diese Passage auf und legt sie im Kapitel "Waffenstillstand « Clarisse in den Mund, ${ }^{20}$ die damit gegenüber Walter ihre Theorie der Doppelwesen rechtfertigt. Durch diese Theorie ist Clarisses Wahn überdies nicht nur strukturell, sondern auch inhaltlich den partizipativen Vorstellungswelten der sogenannten »Primitiven« verwandt: Die Doppelwesen werden, wie im primitivistischen Denken, als symbolische Konstruktionen wörtlich genommen, als Partizipationen oder Metamorphosen gedacht, und der Mensch auf dem Weg von einem animalischen zu einem göttlichen Sein gesehen. Zudem praktiziert Clarisse ein »magisches« Denken, wenn sie z. B. aus Konstellationen von Tieren oder Farben Andeutungen über und Anweisungen für die Zukunft liest, oder wenn sie Namensmagie bemerkt und betreibt. Entspre-

$17 \mathrm{KA} /$ Transkriptionen/Mappe V/4/205.

18 Im Unterschied zu Moosbrugger ist die Forschungslage zum wissenschaftsgeschichtlichen Hintergrund der Clarisse-Figur noch sehr überschaubar. Vgl. aus jüngerer Zeit: Silvia Bonacchi: Die Gestalt der Dichtung. Der Einfluss der Gestalttheorie auf das Werk Robert Musils. Bern I998, S. 249-259. Auf Symptome von Clarisses Geisteskrankheit und z. T. auch in Bezug auf psychiatrische Fachliteratur geht ausführlich ein: Gislind Erna Pietsch Pentecost: Clarisse. Analyse der Gestalt in Robert Musils Roman Der Mann ohne Eigenschaften. Purdue University I990, S. I2-44. Die Darstellung bleibt aber weitgehend nacherzählend; der Bezug auf die Psychiatrie ist nicht systematisch und wird auch nicht reflektiert.

19 Eugen Bleuler: Lehrbuch der Psychiatrie. Berlin I923, S. 35.

$20 \mathrm{KA} /$ Transkriptionen/Mappe I/5/6. 
chend bezeichnet sie sich mehrfach als Hexe: »Thessalische Hexe dünke ich mich! schrie sie in den Aufruhr « eines (von ihr vermeintlich verursachten) Gewittersturms. ${ }^{2 I}$

\section{5 .}

Zweitens: Primitivismus der Musik. In einem Entwurf der Zwillingsschwester-Phase kritisiert Anders, der wie sein Nachfolger Ulrich eine sehr ambivalente Einstellung zur Musik hat, Musiker als »primitive Menschen«: »Primitive Menschen, seid Ihr Musiker. Welche subtile, noch nie dagewesene Motivation wäre nötig, um unmittelbar nach stillem Insichgehn einen tosenden Ausbruch möglich zu machen! Ihr macht es mit fünf Tönen![ ] / Das verstehst Du nicht, Anderle, - lachte Clarisse. [...] Du warst nie krank." (MoE, S. I7I4) Musizieren, Primitivität und Krankheit werden hier aufgrund der ihnen gemeinsamen emotionalen Sprunghaftigkeit in einen unmittelbaren Zusammenhang gebracht. Geht man diesem Hinweis nach, ergibt sich in der Tat eine genealogische wie symptomatische Nähe des manisch-primitivistischen Denkens Clarisses zur Musik. ${ }^{22}$ Die frühesten Anzeichen zu ihrer Ideenflucht zeigen sich, während sie Klavier spielt: »In flatternden Nebeln sprangen Bilder auf, verschmolzen, überzogen einander, verschwanden, das war Clarisses Denken« (MoE, S. I44). Die Flüchtigkeit dieses Denkens folgt musikalischen Gesetzen: »die Musik hielt keinen Augenblick still« (MoE, S. I47). Ebenso entspricht die Verlagerung der Bilder vom zeitlichen Nacheinander in die Gleichzeitigkeit - »oft waren mehrere Gedanken gleichzeitig ineinander da" (MoE, S. I44) - der Schichtung der Motive in mehrstimmiger Musik; auch der Eindruck einer ständigen Metamorphose und eines Ineinanderverschwimmens der Bilder wird den laufenden Modifikationen gerecht, denen die Motive in einer auf Entwicklung und Fortspinnung ausgerichteten Musik unterliegen. Zugleich hat die Ideenflucht mit der Instrumentalmusik eine Tendenz zur Inhaltslosigkeit gemein. Zwar wechseln im manischen Denken lauter Gedanken miteinander ab, doch ist der Inhalt dieser Gedanken unwichtig für den ideenflüchtigen Vorgang. Auch die für die Manie typischen raschen Affektwechsel Clarisses finden ihr Vorbild wie gesagt in der Musik: »Diese Übergänge von lieblich, leise, sanft zu düster, heldisch u[nd] brausend, welche die Musik binnen einer Viertelstunde ein paarmal vollzieht« (MoE, S. I7II). Dass Clarisses Wahnsvorstellungen eine musikalische Qualität haben, zeigt sich auch an ihrem synästhetischen Charakter. In der Beschreibung ihres »anderen Zustands" heißt es: »alles geht ins Musikali-

21 KA/Transkriptionen/Mappe VII/6/42; siehe auch VII/6/49; VII/6/55.

22 Auf die Rolle der Musik in Bezug auf Clarisse geht ein: Pentecost: Clarisse, S. 73-96. Sie sieht jedoch nicht die Verbindung zwischen Clarisses Umgang mit Musik und ihren "primitivistischen«, manisch-schizophrenen Zügen. 
sche und Farbige und Rhythmische« (MoE, S. 659 f.); ebenso beschreibt sie ihre neue Fähigkeit als »[H]ör-[S]ehe[n] [...] eine[r] Welt, in der die Dinge stehen und die Menschen gehen, so wie du sie immer kennst, aber tönendsichtbar « (MoE, S. 7I2). Vorstellungen, die in ihrem Wahnsystem eine große Rolle spielen, sind daher mit musikalischen Phänomenen verbunden. Sie bezeichnet zum Beispiel Moosbrugger, auf den sie in der durch Klaviermusik induzierten Ideenflucht stößt, wiederholt als musikalisch (z. B. MoE, S. 2I7, 435). Im späteren Brief an Ulrich knüpft sie diese Verbindung auch an die vermeintliche Musikalität der drei Silben seines Namens (MoE, S. 7I2). Die akustische Dimension von Sprache spielt auch in anderen ihrer Assoziationen eine prominente Rolle, wie zum Beispiel in der Alliteration "SchlangenSchlingen-Schlüpfrig « (MoE, S. I46) während der bereits erwähnten Klavierspielszene und in sprachlichen Kreationen, in denen Semantik zugunsten von Alliterationen, Assonanzen und »wilden Reimen« (MoE, S. 659, I796) zurücktritt. A-semantisch und zugleich musikalisch präsentieren sich auch viele Kommunikationen Clarisses. Der Brief, den sie Ulrich von der Insel schreibt, ist inhaltlich wirr, aber von einem affektgesteuerten »Rhythmus der Erregung zusammengehalten« (MoE, S. 1376). Es ist daher kein Wunder, dass Clarisse auch ein privilegiertes Verständnis der »Tanzrhythmik« der Urvölker zugeschrieben wird (MoE, S. I787).

6.

Drittens: Primitivismus der Sprache. Oesterreich und Bleuler erwähnen zwar eine ganze Reihe sprachlicher Eigentümlichkeiten Manischer und Schizophrener, gehen deren Gesetzen jedoch kaum nach. Das tut lediglich Kretschmer, und auch nur in Bezug auf seine eingehende Auseinandersetzung mit dem Sprachverhalten der sogenannten »Primitiven«. Musil greift diese Beobachtungen auf und erweitert sie noch. Drei Aspekte fallen dabei besonders auf. Zum einen der eigentümliche Umgang Clarisses mit figurativer Sprache: Sie revitalisiert lexikalisierte und konventionelle Metaphern, indem sie diese wörtlich nimmt; ${ }^{23}$ zugleich aktiviert sie ein magisches Potential von Spra-

23 Die Forschung hat dieses Verfahren so gut wie ausschließlich an der Figur Moosbruggers behandelt. Vgl. z. B. Wilhelm Braun: Moosbrugger dances, in: The Germanic Review 35 (1960), S. 2I4-230, hier S. 22of.; Claudio Magris: Musil und die Nähte der Zeichen, in: Wolfgang Freese (Hg.): Philologie und Kritik. Klagenfurter Vorträge zur Musilforschung. München 198I (= Musil-Studien, Bd. 7), S. 177-193, hier S. I89. Aus jüngerer Zeit: Fred Lönker: Der Fall Moosbrugger. Zum Verhältnis von Psychopathologie und Anthropologie in Robert Musils Der Mann obne Eigenschaften, in: Jahrbuch der Deutschen Schillergesellschaft 47 (2003), S. 280-302, hier S. 288 f. Lönker geht jedoch fälschlich davon aus, dass »in der zeitgenössischen psychiatrischen Literatur kaum ähnliche Phänomene beschrieben« werden (S. 289). Dabei übersieht er, wie zentral dieser Aspekt bei Kretschmer ist. Vgl. auch: Eberhard Ostermann: Das wildgewordene Subjekt. Christian Moosbrugger und die Imagination 
che, indem sie den auf diese Weise animierten Sprachbildern eine über die Darstellung hinausgehende Handlungskraft zutraut.

Zum anderen hat Clarisse nicht nur durch ihren Hang zur Gebärdensprache eine Nähe zu phylogenetischen Frühformen der Sprache, sondern vollzieht in den Insel-Entwürfen zudem die Phylogenese der Sprache nach, wie sie sich u. a. bei Kretschmer darstellt.. ${ }^{24}$ Zunächst entwickelt sie eine Sprache auf der Basis von Ding-Symbolen, die nach den Prinzipien der Ähnlichkeit oder der Kontiguität funktionieren, zugleich aber schon soweit symbolisch sind, dass Anders sie immerhin "verstehen lernen" muss (MoE, S. I74I). Die Ding-Symbole wirken wie eine Steigerung von Clarisses Umgang mit Doppelworten, denn sie verfügen nicht nur über eine Doppel-, sondern sogar über eine Mehrdeutigkeit (MoE, S. I743), die zugleich durch die Dinge immer schon an die Materialität zurückgebunden ist. Aufgrund dieser Einheit von Symbol und Ding sind die Ding-Symbole zwar einerseits Medium der Kommunikation mit dem Gegenüber, doch zugleich eine Form der magischen Kommunikation mit den Dingen selbst: "Zwischen ihr und den Dingen bestand ein fortwährendes Zeichenaustauschen und Verständigen, ein Verschworensein, [...] erhöhte Korrespondenz« (MoE, S. I747).

Als nächste Stufe in der Phylogenese der Sprache entwickelt Clarisse eine Bildersprache aus Sandzeichnungen. Geradezu plakativ umgesetzt ist hier das von Kretschmer für die Bildersprache der sogenannten »Primitiven" erarbeitete Gesetz der Stilisierung sowie auch das Gesetz der katathymisch motivierten Verdichtung. Die Magie dieser Bildersprache liegt in ihrer Affinität zu einer narzisstischen Multiplizierung des Ichs: »die Insel bevölkerte sich mit vielen Clarissen [...], es war eine Wollust [...], überall auf sich selbst zu stoßen « (MoE, S. I742).

Schließlich gelangt Clarisse auf der dritten Stufe der Sprach-Phylogenese zur Entwicklung von Worten und sogar von Lyrik: »Cl. begann ihr

des Wilden in Musils Mann ohne Eigenschaften, in: Neophilologus 89 (2005), S. 605-623, hier S. 608 f., mit Bezug auf sprachphilosophische und ethnologische Bezüge auch S. 6ro f.; Robert Krause: Abstraktion - Krise -Wahnsinn. Die Ordnung der Diskurse in Robert Musils Roman Der Mann ohne Eigenschaften. Würzburg 2008, S. IIo-II4. Gerd-Theo Tewilt: Zustand der Dichtung. Interpretationen zur Sprachlichkeit des sanderen Zustands in Robert Musils Der Mann ohne Eigenschaften. Münster 1990, S. I72-I84, hingegen geht auch auf Clarisses Wörtlichnehmen von metaphorischer Sprache ein und stellt heraus, dass sie dadurch die "Vernichtung des Unterschieds« betreibe (S. 173) und ihre Sprache so gewaltsam werde (S. I74).

24 Christian Kassung: EntropieGeschichten. Robert Musils »Der Mann ohne Eigenschaften« im Diskurs der modernen Physik. München 20or (= Musil-Studien, Bd. 28), geht zwar auf die Sprachentwicklung Clarisses ein, erkennt in ihr aber nicht die phylogenetische wieder, sondern beschreibt ihre Stufenfolge aus dem »Blickpunkt physikalischen Wissens" (S. 429-43I). Auch Magris: Nähte der Zeichen, geht kurz auf Clarisses Sprachschöpfungen ein, mit Fokussierung auf der Etablierung bezugloser bzw. radikal kontext- und kodexabhängiger Zeichen (S. 19I), einer stoffbezogenen Sprache ohne Syntaxbezüge (S. 192) und schließlich autonomer Zeichen ohne Objekt (S. 192). 
Leben in Gedichten auszudrücken« (MoE, S. I753). ${ }^{25}$ Sie befreit sich dafür jedoch von der etablierten Sprache und sprengt Begriffe, indem sie zum Beispiel neue Doppelworte erfindet oder syntaktische Zusammenhänge zerstört und die Worte, z. B. durch Ausrufezeichen oder Wiederholungen, daran hindert, sich gedanklich in diese alten Zusammenhänge zurück zu begeben. Das Ergebnis sind asyntaktische Folgen neuer Wortbilder, wie z. B. »Ichrot« (MoE, S. I753). In diesen Gedichten erscheint Clarisse, wie die frühen Entwürfe klar machen, als proto-expressionistische Dichterin. ${ }^{26}$ Damit ist ein weiterer Bezug zum Primitivismus-Diskurs der Zeit hergestellt, denn Kretschmer, aber auch andere zeitgenössische Psychologen bringen Schizophrenie, primitivistisches Denken und Expressionismus in einen phänomenalen wie genealogischen Zusammenhang, der spätestens seit der Blauer-Reiter-Ausstellung auch unter Künstlern topisch war.

\section{7.}

Aus diesen Beobachtungen zu Manie bzw. Schizophrenie, Musik und Sprache ergibt sich zum einen die primitivistische Kontur der Clarisse-Figur und damit die zentrale Rolle, die primitivistische Motive auch im Mann ohne Eigenschaften spielen. Darüber hinaus lassen sich diese Beobachtungen aber auch als Hinweise auf eine primitivistische Ästhetik lesen, deren Kernpunkte eine partizipative Rezeptionshaltung sowie eine auf Anschaulichkeit statt Abstraktion konzentrierte Zeichenhaftigkeit sind, die eben diese Rezeption herausfordert. Zugleich wird deutlich, dass es sich bei dem Attribut des »Primitiven« nur um ein Konstrukt handelt, speist sich doch diese Ästhetik nicht

25 Roger Willemsen: Dionysisches Sprechen. Zur Theorie einer Sprache der Erregung bei Musil und Nietzsche, in: Deutsche Vierteljahrsschrift für Literaturwissenschaft und Geistesgeschichte 60/I (I986), S. I04-I35, erkennt darin das »Programm einer Expansion des geoffenbarten Namens zur Sprache« (S. I28). Es gehe hier um ein »erotisches, mehr noch ein orgastisches Verhältnis zur Sprache« (S. I29), nämlich zu einer Sprache, die zu ihrer Herkunft "aus Bild und Reiz« zurückgekehrt sei (S. I30).

26 Michael Jakob: Von der »Frau ohne Eigenschaften«zum »Mann ohne Eigenschaften«. Anmerkungen zu Clarisse, in: Josef Strutz (Hg.): Robert Musils »Kakanien« - Subjekt und Geschichte. Festschrift für Karl Dinklage zum 8o. Geburtstag. München I987 (= MusilStudien, Bd. I5), S. II6-I33, liest die Clarisse der frühen Entwurfsstufen als Porträt eines »klassischen Schöpfer-Genius« (S. I30), wenn sie eine neue Sprache, Dichtung und Zeichnungen entwirft und den "anderen Zustand « erlebt (S. I24). Im publizierten Roman hingegen ist daraus, wie Jakob betont, bloße Pseudogenialität geworden. Wenn Clarisse in den ersten Entwürfen Nietzsches früher Geniekonzeption und damit Wagner entsprach, ist die Clarisse des Romans, wie Wagner für den späten Nietzsche, "nur noch Epigonin, die Rolle des Geistes fällt dort dem Wissenschaftler und >freien Geist`Ulrich zu« (S. I2I). Zur Positionierung von Clarisses »identifikatorischer Nietzschelektüre zwischen Walters Kulturkonservatismus und Ulrich als »Personifikation « der »Ambivalenz «, die aus der Auflösung alter Werte resultiert vgl. Alexander Honold: Die Stadt und der Krieg. Raum- und Zeitkonstruktion in Robert Musils Roman Der Mann ohne Eigenschaften. München I995, S. 383-409, hier S. 394 f. 
aus ethnologisch fremden Denkformen, sondern ebenso aus musikalischen Verfahren oder den Tropen der Sprache. Clarisse lässt sich auf die Logiken dieser Verfahren und damit auch auf die Gefahr ein, wahnsinnig zu werden. So agiert bereits sie als Expeditionsreisende, die sich von den Objekten ihrer Expedition beeinflussen und zum Selbst-Experiment verleiten lässt. Im Kontakt mit Clarisse schickt Musil aber vor allem sein Alter Ego Ulrich auf eine Expedition ins »Primitivistische«, das sich als entfremdetes Eigenes präsentiert. Damit komme ich zum zweiten Teil meiner These: die Bewegung von der Expedition zum Selbst-Experiment im Mann obne Eigenschaften.

Die »fremde" Clarisse teilt mit Ulrich nicht nur historische und biographische Zeit, Wohnort und soziale Herkunft, sondern ist außerdem Freundin, potentielle Geliebte und ursprünglich sogar Schwester des Protagonisten, letzteres mit Blick auf einen Brief Musils an das Vorbild Clarisses, Alice, in dem er diese als sein "Schwesterlein " anspricht. ${ }^{27}$ Außerdem weisen zahlreiche Clarisse-Passagen auf parallele Interessen, Ansichten und Erfahrungen Clarisses und Ulrichs hin, prominent vor allem in Bezug auf den anderen $\mathrm{Zu}-$ stand. So bemerkt Ulrich über Clarisse: »[U]nd doch hatte sie in ihrem Anfall [...] Aussprüche getan, die manchen der seinen bedenklich ähnlich waren « (MoE, S. 662). Aus dieser Perspektive lässt sich die Figur der Clarisse als ein ausgelagertes Selbst-Experiment Ulrichs verstehen. Diese Deutung wird unterstützt dadurch, dass sich Ulrichs Vorgänger in den Entwürfen der Zwillingsschwester- und der Spion-Phase, ähnlich wie die Forschungsreisenden in den Expeditions-Erzählungen, von Clarisse »anstecken «, d. h. zum SelbstExperiment verführen lässt. In einer ebenso bekannten wie markanten Passage der Insel-Kapitel heißt es dazu: »Délire à deux: Es handelt sich um zwei Menschen, von denen der eine irr ist und der andre prädisponiert zum Irresein. [...] Durch ständigen Kontakt, indem er beständig den Stoß wirrer und regellose Ideen empfängt, gelangt er dahin wie sein Gefährte zu handeln und allmählig stellt sich der gleiche Wahnsinn bei ihm ein« (MoE, S. I796). Je weiter die Entwicklung des Romans voranschreitet, desto mehr verliert sich jedoch diese direkte Beeinflussung Ulrichs durch Clarisse. An ihre Stelle tritt eine eher distanzierte, kritische Sicht, mit der sich das Selbst-Experiment Ulrichs von Clarisse auf Agathe bzw. auf die Geschwisterliebe verschiebt. ${ }^{28}$

$27 \mathrm{KA} /$ Transkriptionen/Mappe IV/3/45I.

28 Zur "Welt der Clarisse« als »antisoziale Parallelverschiebung zu der asozialen Welt der Geschwister « und der darin enthaltenen "Differenz« von »wahnhafter « und "utopischer " Totalität vgl. Roger Willemsen: Das Existenzrecht der Dichtung, S. 3II-3I8, hier S. 3I5. Schon Philip H. Beard: Clarisse und Moosbrugger vs. Ulrich/Agathe: Der »andere Zustand « aus neuer Sicht, in: Modern Austrian Literature 9/3-4 (I976), S. II4-I30, betont die nüchterne Prüfung, die Ulrich und Agathe in Bezug auf den aZ vornehmen, sowie die Suche nach Bezug des aZ zur praktischen Wirklichkeitsebene und die gemeinsame Natur des aZ bei ihnen, in der Beard eine »quasi-wissenschaftliche" Kontrollinstanz gebildet sieht (S. I2o f.). Zudem betont er, dass der aZ bei Moosbrugger und Clarisse mit Isolation und Zwang einhergeht, bei Agathe und Ulrich jedoch zu einem erweiterten Verständnis ihres Lebens und zu grö- 
Inwiefern lässt sich nun auch Musils Schreiben durch Clarisses Primitivismus beeinflussen, d.h. inwiefern folgt es ebenfalls der Bewegung von der Expedition zum Selbst-Experiment? Oesterreich stellt im Anschluss an ein Kapitel, aus dem Musil intensiv exzerpiert, die These auf, dass nicht nur Schizophrene, sondern auch »Romanschriftsteller« für die »Entstehung eines inneren Doppelbewußtseins « prädestiniert seien. ${ }^{29}$ Vor diesem Hintergrund ließe sich Clarisse also als Figuration des Schriftstellers lesen. Ohne Zweifel produziert sie, wie Walter Fanta gezeigt hat, ${ }^{30}$ "Scheinrealitäten « und imaginiert »fantastische Zeichensysteme«; sie findet auch ihre willigen Zuhörer, zunächst die Vorgänger Ulrichs, dann Walter und den General, der Clarisses Erzählvermögen rühmt: "Sie erzählen so plastisch, daß man alles versteht «. ${ }^{3 \mathrm{I}}$ Kann diese Äquivation für Musils Schreiben Gültigkeit beanspruchen? Schauen wir uns zunächst die Clarisse-Kapitel des Romans an. In vielen dieser Kapitel kippt die auktoriale Erzählung in eine personale, sodass über längere Passagen kein Außenblick auf Clarisse mehr verfügbar ist. ${ }^{32}$ Die Erzählung neigt hier zu einem parataktischen und in Bezug auf die Gedankenverbindung außerdem elliptischen Stil - beides laut Kretschmer typisch für das manische Denken. ${ }^{33}$ Eindrucksvoll zeigt sich das z. B. am Beginn des Kapitels »Clarissens geheimnisvolle Kräfte und Aufgaben.» Vergleicht man seine ersten eineinhalb Seiten mit den letzten des vorhergehenden Kapitels, dann fällt die große Anzahl von Absätzen auf, die den

ßerer Herrschaft über sich selbst führt (S. I25-I27). In einer Variation des letzten Punktes heben auch schon Richard E. Hartzell: The Three Approaches to the >other state in Musil's Mann ohne Eigenschaften, in: Colloquia germanica Io (1976/77), S. 204-219, hier S. 217, und jüngst Maximilian Aue: "Pandämonium verschiedener Formen des Wahns? « Vom Wahnsinn und seinen Grenzen in Musils Mann ohne Eigenschaften, in: Primus-Heinz Kucher (Hg.): Literatur und Kultur im Österreich der zwanziger Jahre. Vorschläge zu einem transdisziplinären Epochenprofil. Bielefeld 2007, S. I35-I44, hier S. I39, I4I, die Gewaltsamkeit hervor, die bei Moosbrugger und Clarisse mit den Erfahrungen des aZ verbunden ist bzw. mit dem Versuch der Vereinigung mit einem Gegenüber. Auch Ostermann: Das wildgewordene Subjekt, S. 6rg ff., betont, dass der aZ bei Agathe und Ulrich im Zeichen wechselseitiger Anerkennung, nicht der Isolation oder der Gewalt wie bei Moosbrugger und Clarisse, steht.

29 Konstantin Oesterreich: Die Phänomenologie des Ich in ihren Grundproblemen. Leipzig I9Io, S. 449 .

30 Walter Fanta: Die Spur der Clarisse in Musils Nachlass, in: Musil-Forum 27 (200I/02), S. 242286, hier S. 284 .

31 KA/Transkriptionen/Mappe V/4/2I2.

32 Insofern folgt er Clarisses Diktum, dass man zum Verstehen eines Menschen ihn "mitmachen« müsse. Das gilt, wie Payne schreibt, auch für das Erzählen über Moosbrugger (Philip Payne: Musil erforscht den Geist eines anderen Menschen - zum Porträt Moosbruggers im Mann ohne Eigenschaften, in: Literatur und Kritik II (1976), S. 389-404, hier S. 392-396). Er hebt die Lebhaftigkeit und den Vorstellungsreichtum des Berichts hervor sowie den Metaphernreichtum und den Rhythmus des Textes, durch den dem Leser die Gefängnismonotonie auf sprachlicher Ebene erfahrbar wird. Aus der grammatischen Disziplin des Textes und der vom Erzähler bekundeten mangelnden Verlässlichkeit der Berichte Ulrichs ergibt sich für Payne aber auch eine Distanzierung des Lesers vom Text und damit auch von Moosbrugger.

33 Ernst Kretschmer: Medizinische Psychologie. Stuttgart ${ }^{12}$ I963, S. I48, I5I. 
linken Rand des Drucktextes optisch zerflattern und das elliptische Denken so gewissermaßen sichtbar werden lassen. Im ersten, vergleichsweise langen Absatz fällt außerdem die Neigung zur Parataxe auf. Acht von zehn Sätzen bzw. Teilsätzen sind kurz und parataktisch konstruiert - ein auffälliger Gegensatz zur letzten Seite des vorhergehenden Kapitels, auf der kein einziger Satz so gebaut ist.

Des Weiteren neigt die Erzählung in diesen Clarisse-Passagen stark zum Einsatz von Metaphern, die, wenn sie wörtlich genommen werden, das assoziative Ordnungsprinzip des Textes darstellen. Dies gilt jedoch - und das ist wichtig - nicht nur für personale Erzählsequenzen, sondern auch für auktoriale, in denen von Clarisse nur die Rede ist. Zum Beispiel wird Clarisses Denken vom Erzähler als ein >Aufspringen und Verschmelzen von Bildern in flatternden Nebeln veranschaulicht sowie ihre Gedanken als shinter einer Bühne stehende Dämonen< (MoE, S. I44) beschrieben. Hochstätter hat auch auf die folgende Passage hingewiesen: ${ }^{34}$ „Clarisse, im langen, die Füße bedeckenden Nachthemd wie ein kleiner Engel anzusehen, stand aufgesprungen im Bett und deklamierte mit blitzenden Zähnen frei nach Nietzsche [...]; im Halbdunkel des Schlafzimmers war das ganz grausig anzusehen gewesen « (MoE, S. 368). Das Wort "aufgesprungen« ist hier offenbar doppelsinnig eingesetzt. Nicht nur meint es das schnelle Aufstehen, sondern auch das plötzliche Auseinanderklaffen einer Persönlichkeit in Engel und Bestie (»blitzende Zähne«), auf das sich das »Grausen« des Betrachters bezieht. Zugleich nimmt der Erzähler damit ein Wort wieder auf, das er in der zuvor zitierten Passage zur Charakterisierung von Clarisses Gedanken verwendet hatte und das insofern gewissermaßen vorcodiert ist. Clarisses Körperhaltung ist so einmal mehr als die Verkörperung eines mentalen Vorgangs lesbar (MoE, S. 366).

Doch nicht nur in den Clarisse-Kapiteln, auch in anderen Teilen des Romans, die keinen inhaltlichen Bezug zu Clarisse haben, zeigt sich $\mathrm{Mu}-$ sil von diesem Merkmal manisch-primitivistischen Sprechens beeinflusst. Bekanntermaßen basieren Ulrichs ebenso wie des Erzählers Versuche, Augenblicke des »anderen Zustands « zu beschreiben, auf Gleichnissen. ${ }^{35} \mathrm{Im}$ Nachlasskapitel »Beginn einer Reihe wundersamer Erlebnisse« wird ein solcher Augenblick mit der Existenzform siamesischer Zwillinge und zugleich mit einem Gleichnis selbst verglichen: »schattenhafte[ ] Vereinigung, von der sie zuvor wie in einem schwärmerischen Gleichnis genossen hatten " $(\mathrm{MoE}$, S. I083). Die siamesischen Zwillinge fungieren gewissermaßen als Gleichnis

34 Auf die "Vielsinnigkeit« dieses Wortes weist schon Dietrich Hochstätter hin: Sprache des Möglichen. Stilistischer Perfektionismus in Robert Musils Mann obne Eigenschaften. Frankfurt a. M. I972, S. I3I.

35 Musil verwendet den Begriff "Gleichnis« als Synonym auch für andere Tropen: "Sinnbild, Gleichnis, Bild, es geht ineinander über«, konstatiert Ulrich im Mann ohne Eigenschaften (MoE, S. I345). 
auf ein Gleichnis, das als solches Gleichnis für den begrifflich nicht fassbaren Augenblick des »anderen Zustands « ist. ${ }^{36}$ Das heißt: Die Erfahrung des "anderen Zustands« lässt sich nicht nur in einem zwillingshaften Gleichnis beschreiben, sondern sie ist zugleich Erfahrung des Gleichnisses. So heißt es an anderer Stelle auch: »Gleichnis als zweiter Zustand «. ${ }^{37}$ Das aber bedeutet, dass es beim Gleichnis gar nicht primär um eine Darstellung des »anderen Zustands« geht, sondern mehr noch um seine Herstellung. Das Gleichnis zu rezipieren, heißt, einen Augenblick des »anderen Zustands « zu erleben. ${ }^{38}$

Auch an Musils Schreiben lässt sich also die Bewegung von der Expedition ins Selbst-Experiment verfolgen. Er wird von Clarisses Primitivismus insofern beeinflusst, als sein Schreiben Züge annimmt, die im Roman und seinen Kontexten als "primitiv« codiert werden: Es verfährt parataktisch, elliptisch und ist vor allem geprägt durch Metaphern und Gleichnisse, und in Bezug auf letztere eignet es sich sogar die performative Magie der Darstellung als Herstellung an.

36 Auf die Verschränkung von Gleichnis- und Zwillingshaftigkeit wird in der Forschung eingegangen, z. B. schon bei Jürg Kühne: Das Gleichnis. Studien zur inneren Form von Robert Musils Roman Der Mann ohne Eigenschaften. Tübingen 1968, S. 155-166, der sich dem Gleichnis der zwei Bäume in Bezug auf die Zwillinge widmet; ebenso bei Tewilt: Zustand der Dichtung, S. I32-I7I, auch bei Wolfgang Riedel: Robert Musil: Der Mann ohne Eigenschaften, in: Dorothea Klein, Sabine Schneider (Hg.): Lektüren für das 2I. Jahrhundert. Schlüsseltexte der deutschen Literatur von I200 bis I990. Würzburg 2000, S. 265-285, der auf die Übertragung des Zwillingsverhältnisses auf Denken und Sprache durch Ulrich hinweist: »Durch sie [Tropen und Metaphern, N. G.] werden, so Ulrich, gleichsam Zwillingsverhältnisse zwischen den Dingen geschaffen" (S. 279). Dabei zieht Riedel korrekt die Verbindung zu Theorien »mythischen« oder »archaischen« Denkens bei Vischer und LévyBruhl oder des Traum-Denkens bei Jung und Freud (S. 279). Der zweite Aspekt, die Verschränkung von Gleichnis und anderem Zustand, wird in der Forschung jedoch lediglich angedeutet und in ihren Implikationen nicht weiter verfolgt, z. B. bei Willemsen: Dionysisches Sprechen, S. II7, sowie Jutta Heinz: Grenzüberschreitung im Gleichnis. Liebe, Wahnsinn und »andere Zustände« in Robert Musils Mann ohne Eigenschaften, in: Dorothea Lauterbach, Uwe Spörl, Uli Wunderlich (Hg.): Grenzsituationen. Wahrnehmung, Bedeutung und Gestaltung in der neueren Literatur. Göttingen 2002, S. 235-256, hier S. 254 f.

$37 \mathrm{KA} /$ Transkriptionen/Mappe II/ $3 / 54$.

38 Das lässt sich mit den Ansätzen zu neuer Ästhetik unterstützen. Die Rezeption eines Gleichnisses beschert das Erlebnis eines aZ, weil, wie in den Ansätzen in Bezug auf den aZ formuliert, hier die Formelhaftigkeit eingefahrener Begriffe gesprengt wird: »In ihm [im Vorgang der Ausdehnung und Zusammenziehung unseres geistigen Seins, N. G.] hat die Kunst die Aufgabe unaufhörlicher Umformung und Erneuerung des Bildes der Welt und des Verhaltens in ihr, indem sie durch ihre Erlebnisse die Formel der Erfahrung sprengt; [...] am aggressivsten und direktesten macht es die Literatur, weil sie unmittelbar mit dem Material der Formulierung selbst arbeitet" (GW 8, S. II53). 
8.

Trotzdem lässt sich Musils Erzählen nicht in diesem Sinne als "primitivistisch « bestimmen. ${ }^{39}$ Denn die Clarisse-Passagen bleiben von Anfang an nicht ohne Kommentierung. In den frühen Entwürfen kommentiert der auktoriale Erzähler Clarisses Denken und Handeln direkt. In den späten Entwürfen werden diese Kommentare zu indirekten, indem sie in Dialoge umgewandelt, oder in Reflektionen Ulrichs bzw. Überlegungen essayistischer Art verschoben werden. Das 38. Kapitel, das so gut wie ganz Clarisses Denken, vorwiegend aus personaler Erzählhaltung, gewidmet ist, wird zum Beispiel abgelöst vom essayistischen 39. Kapitel, in dem der Erzähler und Ulrich über eine »Welt von Eigenschaften ohne Mann« reflektieren, deren Beschreibung sich, wie Simon Jander gezeigt hat, als Reflexion über Clarisses Denken lesen lässt. ${ }^{\circ}$ Diese Verflechtung von gleichnishafter Sprache und Reflexion gilt erst recht für die Passagen über den »anderen Zustand«, in denen sie zudem programmatisch ist. Ulrich fordert, in seiner Abgrenzung sowohl von Clarisse wie von Agathe, bekanntermaßen ein »wirkliche[s] Verstehen« und eine »exakte [Er]forschung « des »anderen Zustands"statt irrationalistischer Schwärmerei; er wendet sich gegen dessen Entgegensetzung zum Denken und will ihn stattdessen als eine »eigentümliche Veränderung des Denkens" begreifen (MoE, S. 575) ${ }^{4 \mathrm{I}}$

39 Regressiven Bewegungen - das ließe sich auch an der Thematisierung von Kindheit und Mystik bzw. Neomystik zeigen - wird im Mann ohne Eigenschaften letztlich stets mit Desillusionierung und Ernüchterung begegnet. Aufgrund dieser regressionskritischen Haltung Musils ist es irreführend, Musils Roman einen "Salto rückwärts in den Mythos« zuzuschreiben, wie das Riedel tut: »[D]er Roman läßt keinen Zweifel daran, daß die Wiedergewinnung dieses Paradieses ohne Regression nicht zu haben ist« (Riedel: Robert Musil, S. 278). Darauf weist nachdrücklich Norbert Christian Wolf hin, indem er die oben zitierte Formulierung Riedels kritisch reflektiert: Norbert Christian Wolf: Salto rückwärts in den Mythos? Ein Plädoyer für das 'Taghelle in Musils profaner Mystik, in: Wiebke Amthor, Hans R. Brittnacher, Anja Hallacker (Hg.): Profane Mystik? Andacht und Ekstase in Literatur und Philosophie des 20. Jahrhunderts. Berlin 2002, S. 255-268. Wolf weist u. a. auf das Scheitern der ParadiesVersuche des Romans hin, auf Musils Distanzierung von der (Neo-)Romantik, auf die "gleiche ontologische Wertigkeit« des ersten und des zweiten Zustands, die Musil beide als begriffliche Instrumente zur »anthropologischen Erkenntnis« in der heutigen Welt dienen (S. 264).

40 Simon Jander: Die Ästhetik des essayistischen Romans. Zum Verhältnis von Reflexion und Narration in Musils Der Mann obne Eigenschaften und Brochs Hugenau oder die Sachlichkeit, in: Zeitschrift für deutsche Philologie I23/4 (2004), S. 527-548, hier S. 533.

41 Und diese Verflechtung von gleichnishafter Sprache und Reflexion gilt ebenso für die essayistischen Kapitel. Das bekannte Kapitel über den Essay gleitet zum Beispiel im letzten Absatz in eine narrative Passage über, die von einem nächtlichen Gartenspaziergang Ulrichs erzählt. In dieser Passage wird auch eine Metapher aufgegriffen ('nebliger Milchschaum`), die schon zwei Seiten zuvor in der Reflexion über das Gebiet des Essays eine Rolle gespielt hatte ('besänftigende Muttermilch`) (MoE, S. 257, 255). In diesem Sinne lässt sich der Essay nicht als Entgegensetzung, sondern nur selbst schon als Verflechtung von Narration, gleichnishafter Sprache und Reflexion verstehen. Vgl. dazu Birgit Nübel: Robert Musil - Essayismus als Selbstreflexion der Moderne. Berlin 2006, die den Essayismus Musils sowohl als "andere Ver- 
Die an Clarisse orientierte Form des »primitivistischen« Erzählens wird also konterkariert durch die reflexiven Passagen des Romans. Damit kommt ein anderes Verständnis dessen ins Spiel, was ein am Vorbild des sogenannten »Primitiven « gewonnenes Erzählen für Musil bedeuten könnte. Es geht gerade nicht um eine bloße Mimesis ans sogenannte »Primitive«, wie es das "primitivistische« Erzählen im Clarisse-Modus ist. Dann hätte man es mit primitivistischer Ästhetik im oben beschriebenen und von Ulrich kritisch reflektierten Sinne zu tun. Sondern Musil nimmt sich die Dichtung der sogenannten Naturvölker auf andere Weise zum Vorbild. Im Zusammenhang mit seinem Essay Literat und Literatur macht sich Musil ausführliche Notizen zu einem ethnologischen Vortrag Hornbostels und betont dabei zwei Aspekte: ${ }^{42}$ Die Dichtung der sogenannten Naturvölker ist ein Ritualgesang, der nicht ein Geschehen darstellen, sondern es herstellen soll. Notwendig dafür ist nicht nur ein ganz bestimmter Inhalt, sondern auch eine ganz bestimmte Form, die durch den Verlauf des herzustellenden Geschehens, das zugleich der Inhalt ist, gegeben ist. Im Anschluss reflektiert er darüber, welches Potential diese Eigenschaften für die gegenwärtige Literatur bergen könnte, und kommt erstens zu der Einsicht, dass auch heutige Literatur noch etwas herzustellen habe, nämlich eine »bestimmte Art von Geist «. ${ }^{43}$ Zweitens konstatiert Musil, dass dem heutigen Dichter für »nicht-ratioide Aussagen« die Formsprache fehle ${ }^{44}$ und es daher gälte, eine solche zu entwickeln. Daraus lässt sich ein Verständnis der von ihm angestrebten »bestimmten Art von Geist « gewinnen. Es handelt sich um eine Formsprache für nicht-ratioide Aussagen, die als solche auf eine Vergeistigung des Körperlichen zielt, wie es der Aufsatz mit Bezug auf den Gestaltbegriff entwickelt. Sie ist das genuin moderne Ziel gegenwärtiger Literatur, welches diese von der Dichtung der sogenannten Naturvölker unterscheidet. Diese Sprachform aber kann nicht identisch sein mit dem Nicht-Ratioiden selbst, sondern muss in aller Sensibilität zugleich dessen Reflexion leisten. Das realisiert der Mann ohne Eigenschaften in seiner Verschränkung von »primitivistischem « Erzählen im Clarisse-Modus und essayistischem Erzählen. Wenn Musil in Literat und Literatur zugleich davon spricht, dass der Zauber der Literatur heute zunächst einmal in sich selbst wirke, so ist damit kein Verzicht auf das Herstellen gemeint. Sondern Literatur stellt sich selbst her als eine Form, in der sich über Nicht-Ratioides geformt sprechen und denken lässt. Entsprechend thematisiert der Roman die Suche nach einer Formsprache des Nicht-Ratioiden nicht nur, sondern ist im Sinne der Herstellung zugleich schon diese Formsprache. Das ließe

nunft« sowie als »immer gleichzeitig im Erlebnis und in der Reflexion« (Kapitelüberschriften des Buches) beschreibt.

42 Zu Musils Lektüre Hornbostels im Kontext des Essays Literat und Literatur vgl. auch Bonacchi: Gestalt, S. 292-30o.

$43 \mathrm{KA} /$ Transkriptionen/Mappe VI/3/4I.

$44 \mathrm{KA} /$ Transkriptionen/Mappe VI/3/II8. 
sich, mit Bezug auf den Literat und Literatur-Aufsatz, als primitivistisches Erzählen im modernen Sinne begreifen. Die Bewegung von der Expedition zum Selbst-Experiment löst sich hier von der Problematik der regressiven Mimesis, die von Musil als primitivistisch kritisiert wird, und führt zum SelbstExperiment als reflektiertem, gegenwartsbezogenen Umgang mit dem entfremdeten Eigenen. 